\title{
THE SURVEY OF CULTURAL HERITAGE AFTER AN EARTHQUAKE: THE CASE OF EMILIA -LOMBARDIA IN 2012
}

\author{
A. Adami ${ }^{\text {a }}$, S. Chiarini ${ }^{\text {a }}$, S. Cremonesi ${ }^{\text {a }}$, L. Fregonese ${ }^{\text {a }}$, L. Taffurelli ${ }^{\text {a }}$, M. V. Valente ${ }^{\text {a }}$ \\ ${ }^{\text {a }}$ Politecnico di Milano, Dept. of architecture, built environment and construction engineering, Via G. Ponzio, 31, 20133 Milan, Italy \\ (andrea.adami, silvia.chiarini, stefano.cremonesi, luigi.fregonese, laura.taffurelli, marco.valente)@polimi.it
}

Commission V, WG V/2

KEY WORDS: Survey, geomatics, earthquake, seismic damage, cultural heritage

\begin{abstract}
:
In recent years many earthquakes hit Italy and its Cultural Heritage. The topic of survey of buildings damaged by seismic events and their interpretation has become very relevant and involved many research groups and Italian Civil Protection.

The damage survey has different roles: in the first stage, immediately after the emergency, the documentation is necessary for the shoring and protection of damaged structures (AEDES forms of Civil Protection). The aim of the second stage is the study and the documentation for the restoration, reconstruction and retrofitting of buildings.

In this context, this study presents methods and instruments used in the survey of 24 churches in the province of Mantua, Lombardy, after the 2012 earthquake sequence. The paper examines the difficulties in surveying damaged buildings and presents the classification used to define, time by time, the most suitable survey approach in the field of Geomatics. In this classification, many aspects are taken into account, such as logistical and practical problems, safety conditions, time preserving methods, economic decisions, complexity of building and required results.

The accurate documentation obtained as a three-dimensional architectural database allows for the observation and analysis of the damage, the definition of interpretative models and the development of intervention projects. Different results are obtained from the point cloud database: traditional 2D representations for architectural projects as well as 3D models for structural analysis or for the development of BIM.
\end{abstract}

\section{INTRODUCTION}

\subsection{The survey after the earthquake}

The effects of the earthquake on cultural heritage can be divided into two large families. The first includes the material effects, i.e. the collapse and damage of the physical structures precisely because of the shock caused by the earthquake and the loss and deterioration of the art pieces, contained in them. The second family of effects, no less serious, is linked to the relationship between the cultural heritage and the community. Often monuments are not only representative of a place or worship, but they are the meeting point for the community. The loss or inability poses to the community the risk of losing its identity. So the reconstruction operations deal not only with the material needs, but alto with the necessity to give back the monument to the community.

This is the context where we worked after the earthquake of Emilia Romagna and Lombardy in 2012. The seismic shocks caused a lot of damage. A large part of cultural heritage, especially the churches in the southern area of Mantua's province, was damaged and required very important structural operations.

In the reconstruction phase, one of the most important evidence was the necessity of a deepened knowledge of the building and of the damages caused the earthquake. The guidelines of the competent authorities confirmed the need for a correct architectural survey and documentation for the structural and restoration project. In this context, Geomatics played an important role as it allowed to use different techniques for the survey and to verify the most suitable ones according to the necessities, the working conditions and the elaborations required for the structural and restoration project.

This article examines the difficulties in surveying the buildings damaged by the earthquake and tries to identify, among the most recent applications in the field of geomatics, the most suitable ones for each case. This research experience regards not only the survey stage, but it describes how acquired data can be used for the projects after earthquake.

\subsection{The earthquake of 2012 in Emilia Romagna and Lombardy}

On 20 May 2012, an earthquake of magnitude 5.9 with epicenter in the town of Finale Emilia caused damage to homes and production facilities in some municipalities of Mantua. On 29 May, the Mantua area was hit again by three successive shocks with a magnitude until 5.8. The earthquakes caused heavy damage to buildings rural and industrial, works of water canals, as well as to buildings and historical monuments and buildings of civil old stone or pebbles. In particular, most of the monuments and places of artistic interest in a wide area, from Mantua to Modena to Ferrara to some municipalities in the province of Bologna, was seriously damaged or partially collapsed (Figure 1).

As most of damaged buildings was composed by churches, the Diocese of Mantua made a detailed report about the effects of the seism. From the initial analysis, the majority of the churches had damages due to the overturning of facades of apses. The churches are particularly exposed to these collapse mechanisms because of their big dimensions, the lack of inner structures to stiffen the structure and often for the bad quality of the connections of the main wall. 


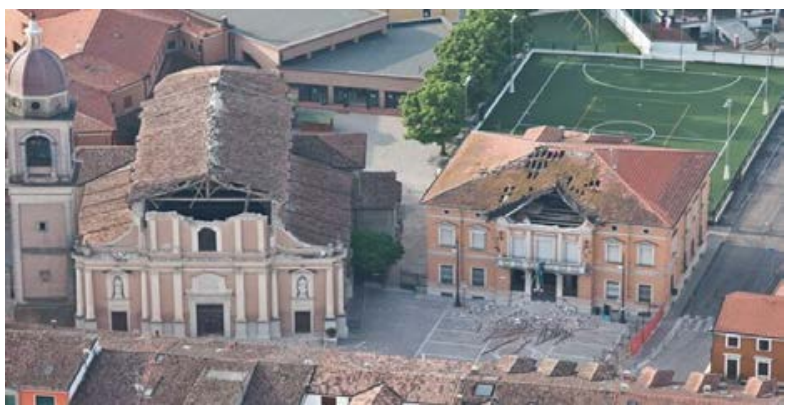

Figure 1. The church of Moglia (near Mantua) and the Municipality seriously damaged after the earthquake.

The churches of the province of Mantua were divided into three classes, according to the seriousness of conditions and the economic damage estimate:

-red code: 10 units, uninhabitable buildings, partially collapsed with danger for public safety and an estimate between 5 and 2 million of euro;

-orange code: 35 units, uninhabitable buildings, partially collapsed with danger for public safety and an estimate less than 2 million of euro;

-blue code: 48 units, uninhabitable buildings, damaged with no danger for public safety and an estimate less than 500.000 euro. In the early stages after the seismic event, the diocese of Mantua asked the Mantua Campus of the Politecnico di Milano, for a cooperation in the survey and analysis of more than 24 buildings (figure 2) among those listed according to the necessity of safety measures or the restoration project.

\section{THE SURVEY}

\subsection{Goals}

We cannot define a single objective for the survey operations as they respond to many different needs. In the post-earthquake stage, we can recognize different purposes.

The first one is to give a general indication of the state of damage of the building to be used in the project of safety measures.

Another purpose is related to the detailed and accurate description of the church. In this case, the survey occurs in a second phase of the post-earthquake, since the temporary structures have already been carried out or were not necessary. Finally, the survey can be also necessary to make interpretative models of the buildings to be used in analytical studies such as structural analysis.

In all these cases, the most useful result of the survey stage is a sort of three-dimensional database made of measurements of the building. It allows to get accurate information about the damage on the architectures and their conditions after the seismic event. It also allows to make new observations and measurements and to integrate the existing ones. The availability of a database 3d, in the form of a point cloud, is also a response to the needs of safety for the operators that can acquire new information without having to go in situ. Point-clouds are suitable for this kind of database as they allows to make measurement, to extracts slices for sections and to build surfaces. Furthermore today point-clouds represent the first result of laser-scanner and photogrammetric survey and they can be integrated in a single reference system.

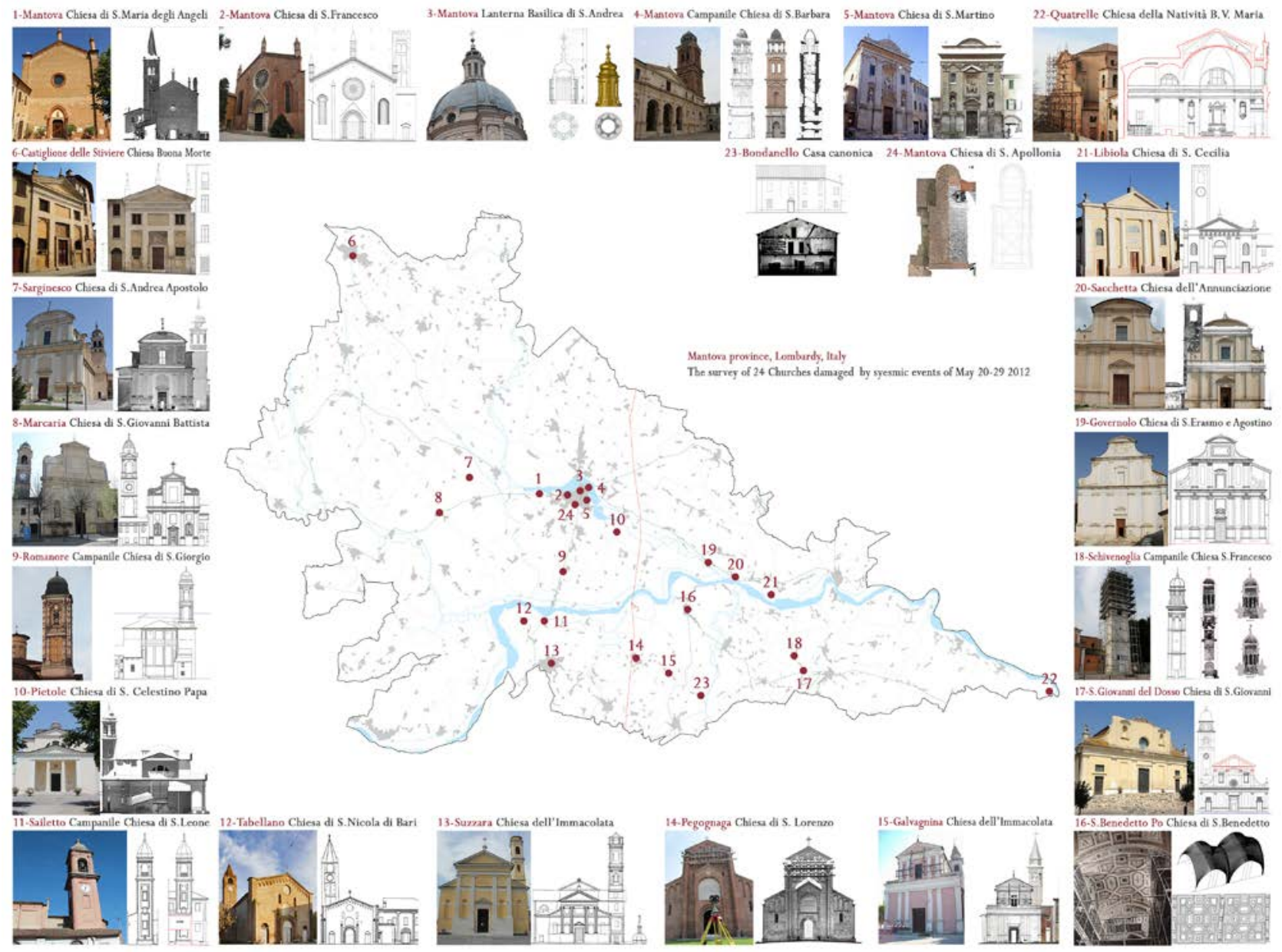

Figure 2. Mantua Province and the 24 buildings surveyed by Hesutech Laboratory of Politecnico 
The $3 \mathrm{D}$ database is also the starting point to obtain the more traditional designs that make up the basis for the restoration project.

Plans, sections and elevations can be extracted through the well-known operation of slicing and by projecting the point cloud on a selected plan. 3D models can be elaborated according to the needs of structural engineers or to be used as the basis of a BIM. Some tests about BIM for restoration were conducted about some churches affected by the earthquake (Achille et al, 2015 b).

The attention of the survey stage has to be focused not only on the geometry of the building, but also on the presence of cracks and their position and dimensions. To understand the new structural configuration of the building it is necessary to locate exactly the cracks and understand if they are only exterior or they regard all the depth of the wall.

\subsection{Difficulties}

The survey after the earthquake presents a number of difficulties or problematic elements related mainly to the operating conditions rather than the technical aspects.

The first difficulty is certainly connected with the safety of the working conditions. In the immediate aftermath of the earthquake, when safety measures have not yet been applied, the biggest risk is the presence of unsafe parts that can break down to successive shocks or stresses of the structure. The risk is very high in churches for the presence of the vaults, especially for the ones next to the façade or the most cracked one. Another safety risk is the presence of collapsed material that prevents access or makes it much more difficult.

Even after the temporary structures, the safety problem remains primarily to the presence of unsafe parts that cannot be reclaimed.

The safety risk, in both cases, involves carrying out the survey activities in a very expeditious and especially prevents, in many cases, to be able to move within the church and reach certain areas. In more severe cases, the surveyor must move within the church under a movable structure, scaffold, which constitutes a sort of safety shell.
The debris and temporary structures are an additional difficulty, as the data acquisition stage becomes much more complex and time consuming. The realization of the topographic network of grading and laser scans are strongly slowed by the presence of scaffolding and this also implies a significant slowdown during the return data.

A particular speech concerns inaccessible parts that still require operator access for relief. It is the case of crawl spaces and raised parts like roofs and bell towers. In many cases we preferred access to those parts through other channels. Where possible we have been used UAV systems for capturing photographic needed to build three-dimensional models, while in other cases the instruments were placed using the platform.

\section{CLASSIFICATION OF SURVEY INTERVENTIONS}

The survey experience of the churches of the Diocese of Mantua, of which this article is about, can be summarized and classified into three different phases. This classification takes into account the various aspects, as said before, that influenced the choices of intervention, methods, tools and number of workers employed. The most relevant are a) the accessibility of places and buildings, b) the time, c) the objectives of the survey and types of graphic restitutions, d) the difficulties inherent to every building, including the geometric complexity and the artistic value.

The Civil Protection, according to their degree of damage and vulnerability, previously evaluated all surveyed churches in the first hours and days after the earthquake. They were defined unusable and coded with the above-mentioned degree of risk (code red, orange, and blue). Areas and buildings attached to the earthquake churches were closed access with security perimeters.

In cases code red, where there were collapses, serious damage to structures and high risk for public safety, the Civil Protection intervened directly to make temporary structures shoring.

In other cases the owners and, in the case of the churches, the Diocese of Mantua, had to work for the safety as well as for the completion of repair/restoration.

The Hesutech group of the Politecnico di Milano has therefore

\begin{tabular}{|c|c|c|c|c|c|c|c|c|c|c|c|}
\hline \multirow[t]{2}{*}{ CHURCH } & \multirow[t]{2}{*}{ PLACE } & \multirow[t]{2}{*}{ CALSSIFICATION } & \multirow[t]{2}{*}{ SURVEY } & \multirow[t]{2}{*}{ TOPOGRAPHY } & \multicolumn{2}{|c|}{ LASER SCANNER } & \multirow{2}{*}{$\begin{array}{c}\text { PHOTOGRAMMETRY } \\
\text { PHOTOSCAN }\end{array}$} & \multicolumn{3}{|c|}{ GRAPHIC PRODUCTS } & \multirow{2}{*}{$\begin{array}{c}\text { APPUCATIONS } \\
\text { 3D- STRUCTURAL ENG }\end{array}$} \\
\hline & & & & & N* SCANS & RGB & & UGHTEN 20 & COMPLETE 20 & $30-8 \mathrm{BIM}$ & \\
\hline SANTA MARIA DEGU ANGELI & ANGELI & 1 & COMPLETE & (9) & 16 & $-\cdot$ & - & (:) & - & & (2) \\
\hline CANONICA & BONDANELLO & 2 & COMPLETE & (1) & & - & - & - & () & & \\
\hline CONCEZIONE BV - BUONA MORTE & CASTIGLIONE D. STIVIERE & 2 & COMPLETE & (ㄹ) & 6 & - & () & - & (2) & & \\
\hline SS. ERASMO ED AGOSTINO 1 & GOVERNOLO & 2 & COMPLETE & (2) & & .. & - & - & ()ㅜㅇ & & (2) \\
\hline S CECIUAA & UBIOLA & 2 & COMPLETE & (4) & & .. & -- & - & (): & & (). \\
\hline SANDREA APOSTOLO LANTERNA & MANTOVA & 3. & COMPLETE & (4) & 10 &. & (4) & - & (*) & & (2) \\
\hline SFRANCESCO & MANTOVA & 3 & PARTIAL-CHAPEL & (2) & 3 &. & - & - & (2) & & (2) \\
\hline SAN MARTINO & MANTOVA & 3 & COMPLETE & (2) & 27 &. & (2) & - & (2) & & \\
\hline CAMPANILE DI SANTA BARBARA & MANTOVA & 3 & BELLTOWER & ()ㅜㅇ & 55 & .- & (). & - & (2) & & (2) \\
\hline SAN GIOVANNI BATTISTA & MARCARIA & 3 & COMPLETE & (2) & 37 & - & (4) & - & (2) & & (2) \\
\hline IMM. CONCEZIONE DELLA BV MARLA & GALVAGNINA & 2 & COMPLETE & (3) & & (:) & - & - & (2) & (ㄱ) & ()ㅜㅇ \\
\hline SLORENZO & PEGOGNAGA & 2 & COMPLETE & (1) & &. & - & - & (9) & & (2) \\
\hline SCELESTINO PAPA & PIETOLE & 1 & COMPLETE & (2) & 16 & -. & - & (4) & - & & (2) \\
\hline NATIVITA' BEATA VERGINE MARIA & Quatreue & 3 & COMPLETE & (1) & 36 & .. & (1) & - & (2) & & (2) \\
\hline CAMPANILE SAN GIORGIO MARTIRE & ROMANORE & 2 & BEU TOWER & (). & 9 & .. & - & - & (2) & & (2) \\
\hline ANNUNCIAZIONE BV MARIA & SACCHETTA & 3 & COMPLETE & (4) & 49 & - & (1) & - & (*) & & (2) \\
\hline CAMPANILE S LEONE MAGNO & SAILETto & 2 & BEL TOWER & (4) & 8 &.- & - & - & (4) & & (2) \\
\hline S BANEDETTO ABATE & SAN BENEDETTO PO & 2 & PARTIAL-VAULTS & - & 9 & .. & - & - & (2) & & \\
\hline S GIOVANNI BATTISTA & SAN GIOVANNI D. DOSSO & 3 & COMPLETE & (2) & 52 & () & - & - & (2) & & (ㄹ) \\
\hline S. ANDREA APOSTOLO & SARGINESO & 1 & COMPLETE & (1) & 16 & .. & -- & (2) & - & & \\
\hline CAMPANILE CHIESA SFRANCESCO & SCHIVENOGUA & 2 & BEL TOWER & (2) & 45 &. & - & - & (2) & & \\
\hline IMMACOLATA CONCEZIONE & SUZzara & 2 & COMPLETE & (2) & &.- & - & - & (2) & & (4) \\
\hline S NICOLA DI BARI & TABELUano & 2 & COMPLETE & (1) & & - & - & - & (2) & & () \\
\hline S. APOLIONIA & MANTOVA & 3 & PARTIAL-ROOF & ()ㅗ & 29 & .. & ()ㅜㅇ & - & (2) & & \\
\hline
\end{tabular}

Table 1. Classification of survey interventions 
worked in collaboration with the Diocese of Mantua, identifying the type of approach according to the situations and demands (table 1).

First, with the managers and the competent authorities, we identified the modalities of access to the buildings and the levels of risk. Then we trained workers as concerning safety and the use of instruments of survey, to work quickly and accurately.

The common element to all applied method was the metric accuracy, the speed of acquisition (to stand inside the buildings as less as possible) and the possibility to obtain the above mentioned 3D database to document the most important disruptions (crack patterns in the order centimeter, out of plumb, separations, etc.).

According to all this need and criteria, the interventions were classified (as in table 1) into different levels of complexity of the actions involved and importance of the damage.

Case 1) low complexity: low-risk people, survey for the implementation of interventions of small or medium-sized, short acquisition time and time processing, topographic network and laser scanner.

Case 2) average complexity: moderate risk, survey for final restoration and seismic improvement of buildings to medium complexity, complete drawings of the building, using topographic network and laser scanner

Case 3) high complexity: high-medium risk, survey for final restoration and seismic improvement of buildings in highcomplexity, full drawings, use and integration of various methods such as laser scanning, photogrammetry from the ground and from above, or UAV aerial platform with a topographic network.

As regards the instrumentation, in general, we decided to use the terrestrial phase shift Leica Geosystem HDS7000 laser scanner, that allows to detect 1 million points per second with a range noise of $0.3 \mathrm{~mm}$ rms at $10 \mathrm{~m}$ ( $80 \%$ white surface). It has also the possibility, very useful in a emergency context, of remote control Wireless LAN.

With this instrument, the exterior and the interior of the buildings were acquired, in various light conditions, generally in the absence of electricity and low light. The single scan last from 3 to 12 minutes and it allows to stay inside buildings for a short time just to set the acquisition and then to stay in a safe place. The laser scanner was managed from safe positions previously identified in areas with lower risk of collapse or created ad hoc for the stop of the operators.

In any case, the reference system of the work was materialized through a topographic network. In this way all measures, also acquired with different methods and tools, refer to a single reference system, and we can have a fully control of precision. The single scans were georeferenced in the above mentioned topographic system through the use of target detected by a total station. This process, based on a topographical support, was very useful when, for lack of access to certain areas, there was no enough overlap for the cloud to cloud orientation.

\subsection{Case 1: low complexity}

Churches classified in this group (Sarginesco, Angeli and Pietole) have a low level of complexity for both the risk and the problematic to be addressed in the operational phase and the amount of documentation to be produced for the design of interventions.
Usually these building had a quite simple architectural configuration. They had a unique nave and the presence of a crack pattern was concentrated in the key structural points. These building were surveyed by laser scanner in a very short time. Time required for the entire survey (acquisition stage, data processing and drawing editing) was about 1 week.

For each church, 16 laser scans (average resolution $3.1 \mathrm{~mm}$ at $10 \mathrm{~m}$ - time required about $6 \mathrm{~min}$ ) were made and they were georeferenced in the topographic network. Usually the entire work was made by three operators.

The processed products were architectural plans and sections. To give more information to the designer, we enriched the classic drawings with the orthographic projection of the point clouds, as a background. These images can be considered as quasi-orthophoto as they have the same characteristic of an orthophoto even if they are not released with images. They are measurable, with a pixel coverage on real object of about $4 \mathrm{~mm}$. They allow a better comprehension of the architecture and to extract the change in the cracks, the gaps, the degradations (figure 3).

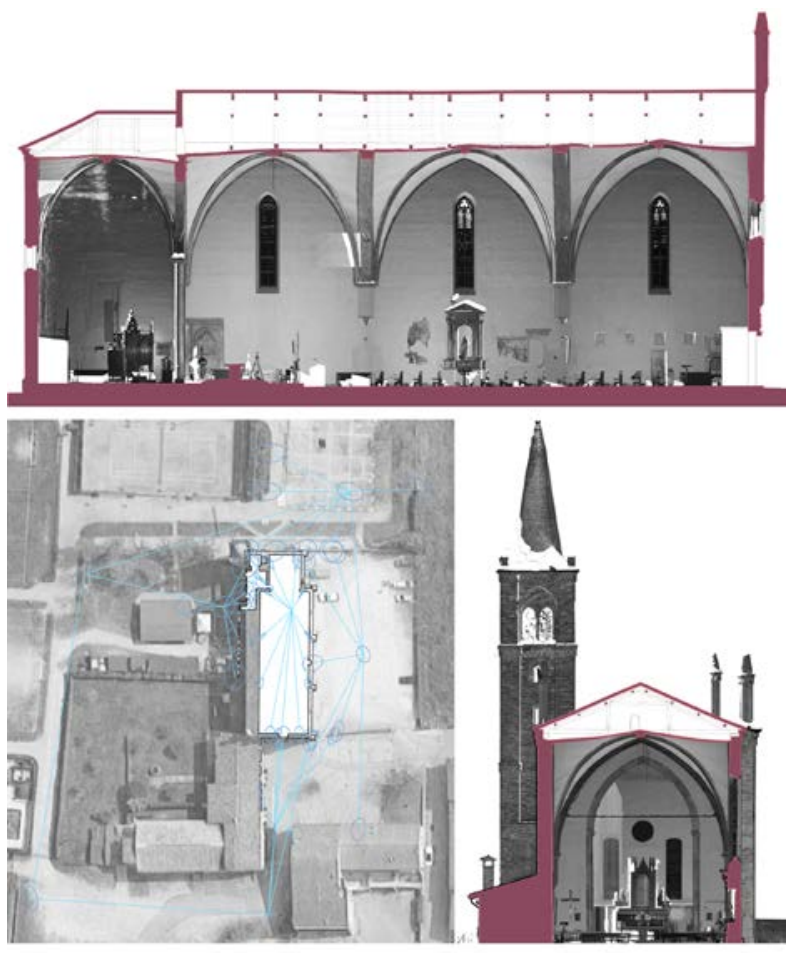

Figure 3. Example of survey of case 1 building. In particular: Chiesa degli Angeli. Above the longitudinal section with the quasi orthophoto on the background. Below, on the left the topographic network, on the right a cross section with the bell tower

\subsection{Case 2: medium complexity}

This category includes the most frequent cases as from table 1 . The purpose was to provide a 3D database as complete as possible of the building to extract key information on the state of damage of the structures, such as misalignments, curvature of the vaults and arches, gaps between wall elements and horizontal structures, major cracks, deformation.

All major 2D representations of the building were required: ground floor plans, sections and elevations (internal and external). In most of the cases, there were no available and reliable surveys, printed or in digital form, which architects and 
engineers could use to design the executive restoration project and seismic strengthening.

The churches of this category were more complex because of their geometries, architectural dimensions and the presence of high detailed decorative apparatuses.

Therefore, it was necessary to acquire a greater amount of scans than in case 1 to get the right level of resolution and overall coverage. We acquired about 32 scans for interior and exterior inside and, where possible, we surveyed the attic between the vaults and the roof.

The medium resolution was $3.1 \mathrm{~mm}$ at $10 \mathrm{~m}$.

Usually three or four operators made the acquisition (laser scanner and topography) in 1 or 2 days. The deadlines to complete the drawings was about days (figure 4).

The elaborations were the same produced for the case 1 buildings: traditional 2D drawings with quasi orthophoto as background.

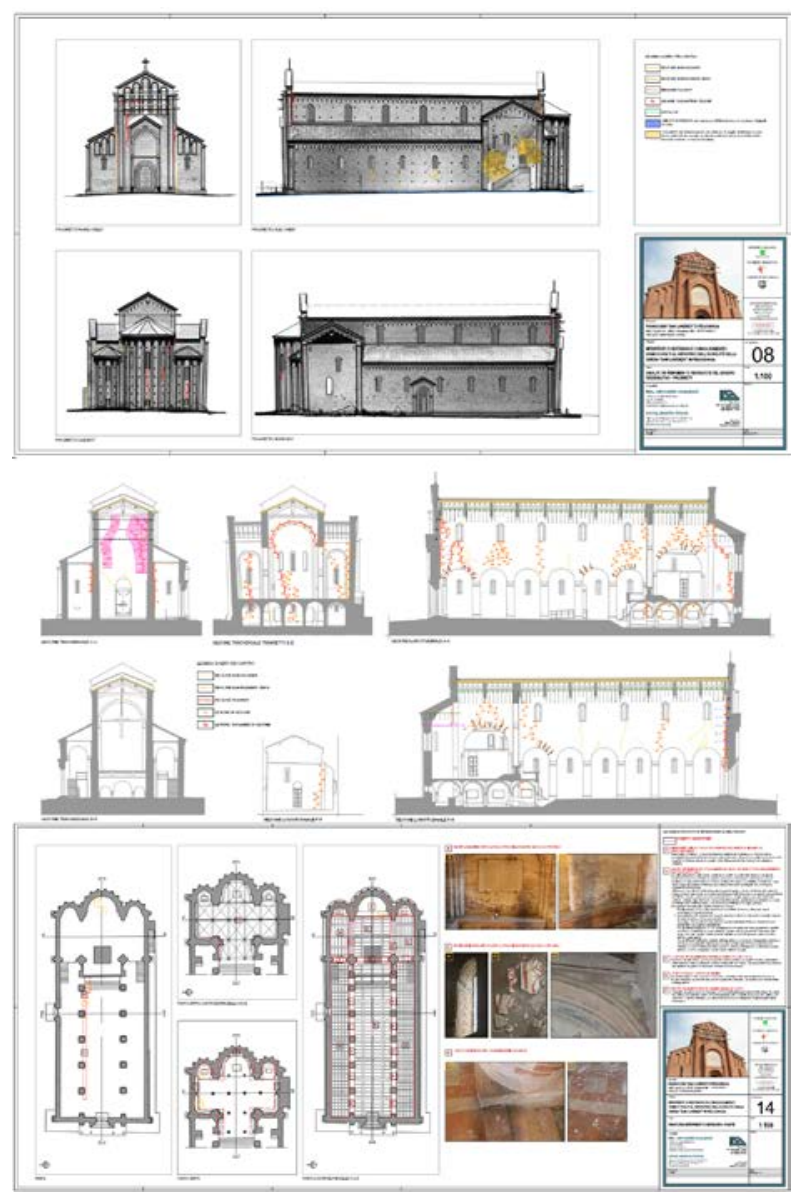

Figure 3. Example of survey of case 2 building. In particular:

San Lorenzo di Pegognaga. Above the drawings from the survey, in the middle the crack analysis based on the orthophoto and below the restoration design.

\subsection{Case 3: High complexity}

The churches of this third group were really complex and important in the area. Furthermore they presented logistic problems determined by the collapse and damage reported after seismic events. For all these reasons in the cases of this group it was necessary to coordinate the survey with the authorities and those involved and a careful planning of the survey. These conditions proved much longer times. In all the churches of the case 3, the Civil Protection intervened only on the facades and on roofs to avoid collapses to the external public spaces. Nevertheless, they did not make shoring or support structures inside the buildings and left a critical situation as after earthquakes.

The churches of this category are characterized by decorations, frescoes and complex geometries to detect. In some cases, such as the bell tower of the church of Santa Barbara (Achille et al, 2015a) or the lantern of the dome of the Basilica of St. Andrew in Mantua, the survey had to take into account the height of the structures ( $\mathrm{h}=53 \mathrm{mt}$ for S. Barbara, 73mt for S . Andrea). In addition, the density of the ancient urban context made it difficult to find good positions on the ground from which to acquire measures. The problems were concerning the measuring angle very foreshortened and the possibility of having a uniform resolution on the architectural surface.

Therefore, for all the above-mentioned reasons, in case 3 the laser scanner acquisition was integrated with a 3D photogrammetric survey based on automated 3D image modeling.

In recent years, many studies showed the results obtained with image-matching and close-range in architectural survey, and compared them with the given laser scanning. The common result is reliability of the photogrammetric approach and the successful integration and/or interchangeability of methodologies (Fassi et al, 2013).

This approach was necessary especially for those parts of buildings, which were inaccessible to other "traditional" methods, since it allows to extract point clouds from the images. Moreover, it gives also the opportunity to have true orthophotos in short times or to produce texture models. In both cases it is possible to obtain information about the damage and injury present, essential for the drafting of the restoration project.

The photogrammetric models obtained by dense image matching were calculated using the software Agisoft PhotoScan. The images were oriented automatically and then we obtained a dense point cloud. This resulting cloud was scaled and rototranslated in the reference system, allowing the integration and comparison with the data laser scanning.

The digital camera used in the surveys from ground and aerial platform (survey of the roof of the church of S. Apollonia in Mantua) is the Canon EOS 5D Mark III (22 Mpixel) with lenses 24 and $35 \mathrm{~mm}$.

The aerial surveys have been performed instead, for the two cases cited Lantern Andrea S (figure 4) and the bell tower of Santa Barbara, with an octocopter equipped with digital camera Canon EOS 650D from 18Mpxl, 2 gyros, GPS and barometric altimeter for the flight control.

In the survey of these churches, we were employed five operators for two days of acquisition. Deadlines to complete the drawings and delivery of the documents with 2D orthophotos: 40 days.

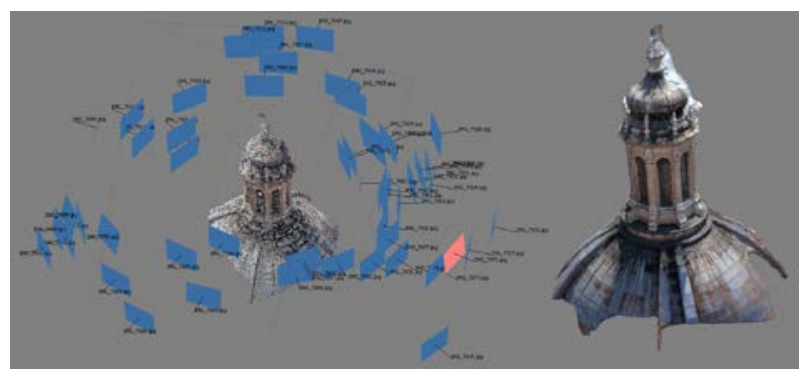


Figure 4. An example of the photogrammetric approach applied for the survey of the lantern of S. Andrea in Mantua. On the left the images oriented and the sparse point cloud, on the right the 3D model

Other churches of this group needed some specific approaches. In many cases, it was necessary to acquire the real RGB values of the surfaces for the documentation of preservation status of frescoes, decorations, furniture elements and altars in stone and marble. The problem of the absence or poor interior lighting has been overcome with the use of lighting balloon for uniform enlighten (balloon Airstar, model 575W Sirocco, hot restrike, with pole to $6 \mathrm{mt}$ and internal ballast) where it was logistically possible to bring electrical connections. In the church of S. Giovanni del Dosso, for example, we used those lighting balloon to avoiding areas at risk of collapse and stopping under movable scaffolding, designed and calculated specifically for the protection of operators. In other cases, we tried to make the most of natural lighting, working in post-production on color management and display of images to their mosaicking (i.e. church of Sustinente - figure 5).

In cases where there were more rigid constraints of movement inside buildings, because of the risk of collapse (as the Church of San Giovanni del Dosso), we decided to acquire data color through the automatic acquisition of spherical panoramas. We used the motorized head CLAUSS Rodeon VR, for controlling the digital camera on the same tripod of laser scanner and to obtain a high resolution panorama (24540x12270 pxls 300dpi, 24-bit depth). The panorama was re-oriented on laser scanner data and the RGB value was associated to laser scanner point clouds, acquired at high density $(1.6 \mathrm{~mm}$ at $10 \mathrm{~m}$ - about 13 mins). From these colored point clouds, we extracted the same quasi orthophoto as explained before with a pixel size on the object of $2 \mathrm{~mm}$.
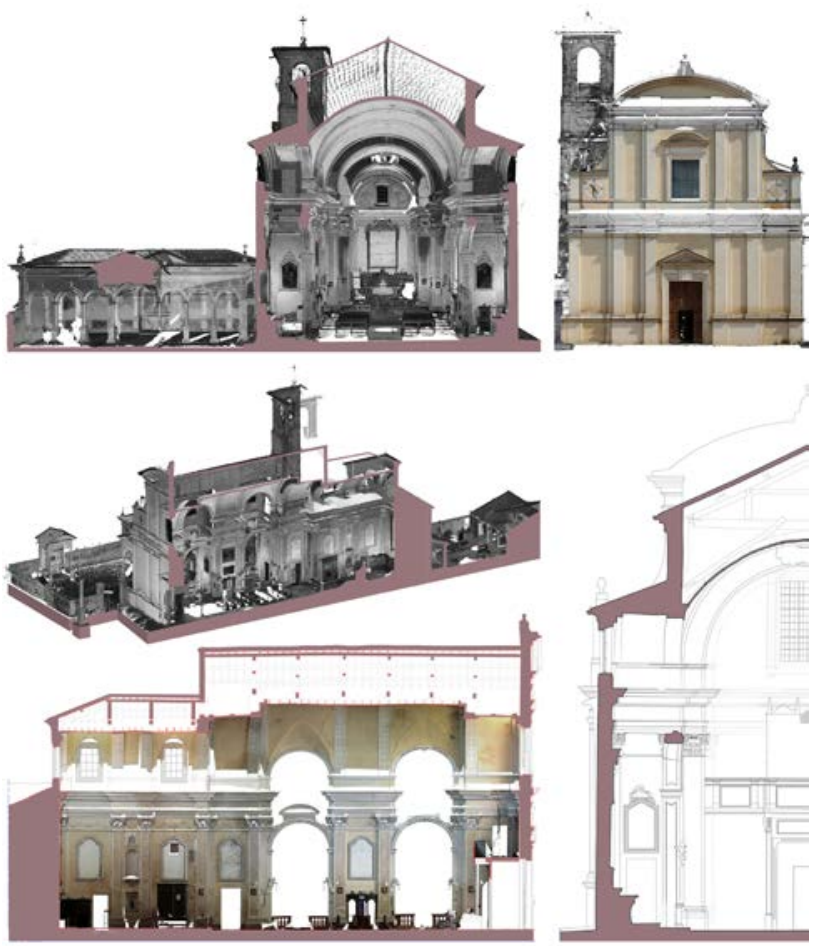

Figure 5. The complete documentation of a church of case 3: church in Sustinente, near Mantua. Above the cross section and the orthophoto of the façade, the 3D model and the cross sections with and without orthophoto

\section{OTHER APPLICATIONS}

\subsubsection{Structural analysis}

An effective seismic vulnerability assessment of the churches can be obtained through non-linear dynamic and static analyses by means of suitable finite element models. To this aim, detailed three-dimensional finite element models of the churches are created through the computer code Abaqus (ABAQUS) taking into consideration geometrical and material nonlinearity. A damage plasticity material model, exhibiting softening in both tension and compression, is adopted for masonry. Figure 6 shows the finite element numerical models of two churches: San Lorenzo Church in Pegognaga and San Giovanni Battista Church in San Giovanni del Dosso. The choice of the element size is done in order to share the advantages of sufficiently reliable results and numerical efficiency during the non-linear dynamic analyses that usually need very long time to be performed.

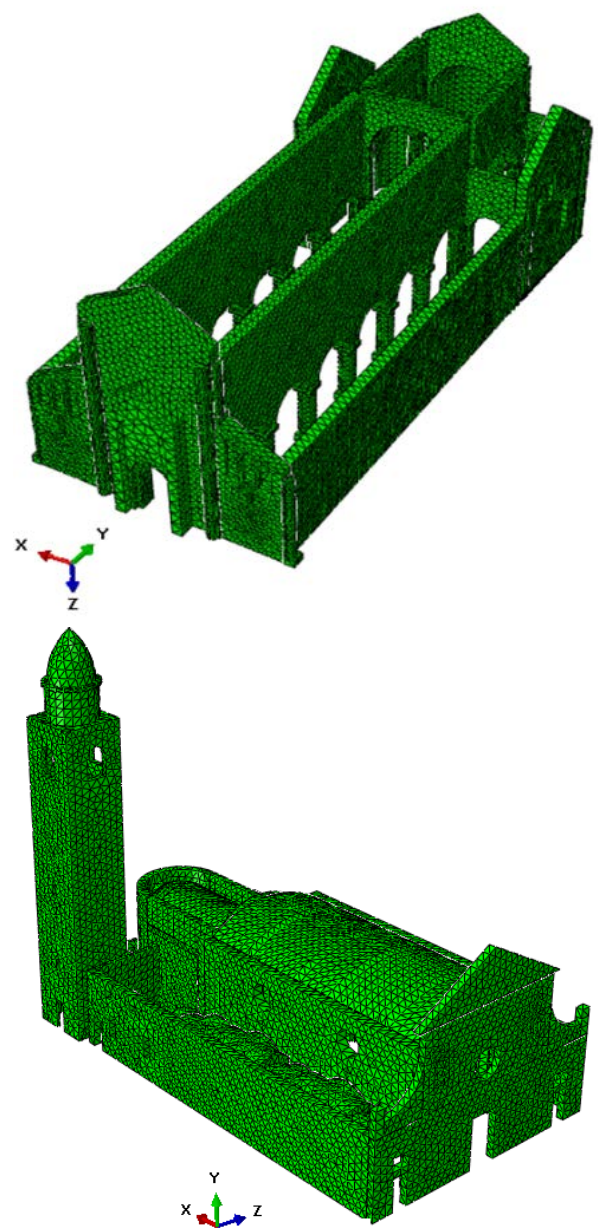

Figure 6. Finite element models of San Lorenzo Church in Pegognaga and San Giovanni Battista Church in San Giovanni del Dosso

In order to investigate the seismic behavior of the churches, 
different types of numerical analyses are performed, including simplified static analyses according to Italian Guidelines on architectural heritage, eigen-frequency analyses, non-linear static (pushover and kinematic limit analysis) and full nonlinear dynamic analyses, (Milani, Valente, 2015a)(Milani, Valente 2015b). The different procedures, affected by different levels of accuracy and complexity, may provide a convincing picture of the weaknesses of the structure and are systematically applied to many churches that suffered damage during the recent 2012 Emilia earthquake sequence. The analyses of several different case studies showed the necessity of a deep knowledge of masonry. In fact the very large geometric variability and irregularity, as well as the architectural complexity, are responsible of the very different behaviors.

A preliminary assessment of the dynamic behavior of the churches can be obtained by performing an eigen-frequency analysis on the three-dimensional finite element models. Albeit approximate, because masonry exhibits a non-linear behavior even at very low levels of seismic excitation, such a standard approach allows identifying the vibration modes characterized by a high participating mass and may give a rough indication of the weaknesses of the structures that can be compared with more sophisticated methods of analysis.

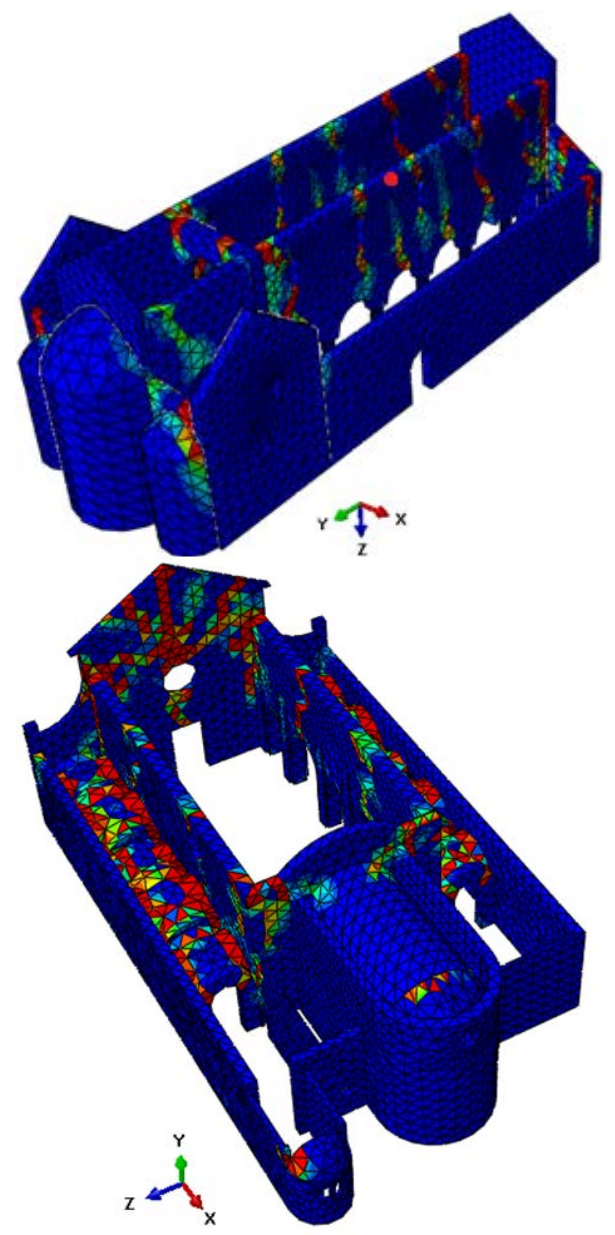

Figure 7. San Lorenzo Church in Pegognaga and San Giovanni Battista Church in San Giovanni del Dosso: false colour representation of tension damage at the end of the numerical simulations.

In order to capture all possible failure mechanisms, non-linear dynamic analyses are conducted applying simultaneously two accelerograms along two horizontal orthogonal directions. Such type of analysis is very time-consuming, but it is much more accurate and reliable than other approaches for its capability to identify in- and out-of-plane, as well as local and global failure mechanisms. The seismic performance assessment of the churches is carried out mainly in terms of displacement timehistory of some control points and tensile damage distribution.

Figure 7 shows the contour plots of tension damage at the end of the non-linear dynamic analyses performed on San Lorenzo Church and San Giovanni Battista Church. The results of the non-linear dynamic simulations show the high vulnerability of the churches under horizontal loads. The role played by the complex geometry, local irregularities and the insufficient resistance of the constituent materials is investigated, also in light of the actual failure mechanisms. In all cases, the numerical analyses provide a valuable picture of all possible failure mechanisms, thus giving useful hints for appropriate and suitable interventions of consolidation to reduce the seismic vulnerability of the churches.

\subsubsection{Modello di Galvagnina per il BIM o di Lanterna di $\mathrm{S}$ Andrea per le opere provvisionali}

All church acquisitions are designed to achieve accuracies and resolutions appropriate to the scale of 1:50 and 1:20, in order to allow a deepen assessment of the actual state of the buildings. In addition to export 2D graphics (plans, sections and elevations), three-dimensional models have been developed for BIM applications (as for Galvagnina Church). In these cases the given three-dimensional laser scanning or photogrammetric, it is essential for the modeling of architectural elements, starting from the actual shape of each after the damage and misalignment due to the earthquake, for a study and a realistic analysis of the overall behavior of the building.

The BIM was projected in order to link to each part of the building some information about the conservation and the restoration project. The entire project, described in (Achille et al, $2015 \mathrm{~b}$ ) focused of the possibility to use a BIM model not only in the project of new buildings, but also in the restoration stage, and in particular for the maintenance plan (figure 8).

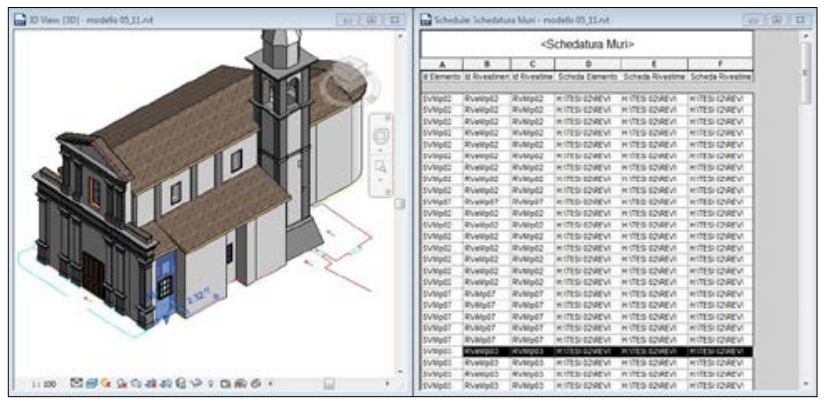

Figure 8. The BIM model of the church of Galvagnina with the linked data of the state of conservation and restoration project.

\section{CONCLUSIONS}

This paper shows how Geomatics can answer to the specific requirements of the survey of buildings damaged by the earthquake. The instruments and methods used in this research are not entirely innovative, and indeed they represent a consolidated approach in the field of Cultural Heritage survey. But this study demonstrates that they can be very useful also in emergency contests. Laser scanner and photogrammetric survey can be selected, or integrated, according to the needs of the 
operations such as safety conditions for the operator, reduce time consuming, extract as much information as possible. The possibility to use these methods from different platforms (the ground, an elevated platform or a UAV) allows to access also the most difficult parts of the building and guarantees the safety for the operators.

The 3D database, which is the first result of both applied method, is something more than a pointcloud as it allows to have more information about the geometry of the structure and its behaviour. And also the 3D database is the starting point for many other analysis connected with the earthquake, from the structural study to the conservation and restoration project.

\section{ACKNOWLEDGEMENTS}

Acknowledgements of support for the project to Diocese of Mantua.

\section{REFERENCES}

ABAQUS ${ }^{\circledR}$, Theory Manual, Version 6.14.

Achille, C., Adami, A., Chiarini, C., Cremonesi, S., Fassi, F., Fregonese, L., Taffurelli, L., 2015a .UAV-Based Photogrammetry and Integrated Technologies for Architectural Applications-Methodological Strategies for the After-Quake Survey of Vertical Structures in Mantua (Italy), in: Sensors 2015, 15(7), pp.15520-15539.

Achille, C., Adami, A., Fassi, F., Fregonese, L., Spezzoni, A., Taffurelli, L., 2015 b. BIM: an integrated model for planned and preventive maintenance of Architectural Heritage. In: Proceedings of 2nd International Congress on Digital Heritage 2015, Granada, Spain.

Fassi F., Fregonese L., Ackermann S., De Troia V., 2013. Comparison between laser scanning and automated $3 \mathrm{~d}$ modelling techniques to reconstruct complex and extensive cultural heritage areas. In: Proceedings of International Archives of the Photogrammetry, Remote Sensing and Spatial Information Sciences, Trento, Italy, February 25 - 26, Vol. 405/W1, pp. 73-80;

Fratus de Balestrini E., Ballarin M., Balletti C., Buttolo V. , Gottardi C., Guerra F., Mander S., Pilot L., and Vernier P., 2013, Survey Methods For Earthquake Damages In The "Camera Degli Sposi" Of Mantegna (Mantova), In Int. Arch. Photogramm. Remote Sens. Spatial Inf. Sci., XL-5/W2, 265270, 2013 doi: 10.5194/isprsarchives-XL-5-W2-265-2013

Milani G, Valente M., 2015 (a). Comparative pushover and limit analyses on seven masonry churches damaged by the 2012 Emilia-Romagna (Italy) seismic events: possibilities of nonlinear Finite Elements compared with pre-assigned failure mechanisms. Engineering Failure Analysis; 47: 129-161.

Milani G, Valente M., 2015 (b). Failure analysis of seven masonry churches severely damaged during the 2012 EmiliaRomagna (Italy) earthquake: non-linear dynamic analyses vs conventional static approaches. Engineering Failure Analysis; 54: $13-56$.

Modena, C., Casarin, F., Da Porto, F., Munari, M., Simonato, E., 2010. Cultural Heritage Buildings and the Abruzzo Earthquake: performance and post-earthquake actions. Advanced Materials Research, Vol.133-134, pp 3-17, TrasThech pubblications, Switzerland. 